\title{
Algorithmic improvements and calibration measurements for flavour tagging at the ATLAS experiment
}

\author{
Marco Battaglia* \\ Santa Cruz Institute of Particle Physics \\ University of California, Santa Cruz, CA (USA) \\ E-mail: marco.battaglia@ucsc.edu \\ Alessandro Calandri \\ CPPM Marseille (France) \\ E-mail: alessandro.calandriecern.ch
}

The identification of jets containing bottom or charm hadrons is crucial to the LHC physics program. Top-quark decays proceed almost exclusively through a $b$-quark. The Standard Model Higgs boson decays predominantly to $b \bar{b}$ pairs. Several scenarios of new physics result in an enhanced production of fermions of the third generation, such as models with an extended Higgs sector or scalar top and bottom quark production in Supersymmetry. These physics scenarios correspond to very different environments for performing the identification of jets with heavy flavour hadrons of very different kinematics. This justifies a special effort in the identification of $b$ - and $c$-jets with the ATLAS detector [1] over a broad kinematical range.

Flavour tagging is based on the reconstructed trajectories of particle tracks and their extrapolation to the colliding beam envelope. The introduction of the ATLAS IBL pixel layer located at a radial distance of $3.3 \mathrm{~cm}$ from the interaction region with a $10 \mu \mathrm{m}$ hit spatial resolution in Run 2 [2] resulted in an improvement of the track extrapolation resolution by a factor up to 2 at low values of the track transverse momentum, $p_{\mathrm{T}}$. The adoption of a stochastic model of energy deposition in Si pixels [3] and of a more realistic description of the material in the ATLAS inner detector system in the 2017 software configuration improved the data/MC agreement for the track extrapolation resolution and the response of track-based taggers.

Improvements and innovations in physics taggers, new approaches to multivariate analysis and training samples have brought optimised and more performant flavour-tagging algorithms for the analysis of the 2017 LHC collision data with ATLAS. This contribution summarises these recent developments. More details on jet flavour tagging in ATLAS and its performance in the 2017 configuration can be found in $[4,5]$.

The European Physical Society Conference on High Energy Physics

5-12 July, 2017

Venice

\footnotetext{
${ }^{*}$ Speaker.

${ }^{\dagger}$ On behalf of the ATLAS Collaboration.
} 


\section{Physics Taggers}

Flavour tagging in ATLAS is performed by combining the response of track-, particle- and vertex-based physics taggers $[4,5]$. The algorithmic improvements to track-based taggers include the use of a Recurrent Neural Network algorithm that processes tracks exploiting spatial and kinematic correlations without reliance on secondary vertex finding (RNNIP). This uses the information from the track-by-track correlations within a jet, providing superior and complementary tagging capability. Its use results in a gain of up to a factor of 2.5 in light-jet rejection at $70 \% b$-jet efficiency compared to the simpler impact parameter-based IP2D and IP3D taggers [6]. The impact parameter-based (IP2D and IP3D) taggers remain complementary to the RNN performance, in particular for $b$-jets with low $b$-hadron charged decay multiplicity.

The introduction of a particle-based, soft muon tagger (SMT), identifying muons from semileptonic decays of $b$-hadrons offers an alternate tagger for jets with semileptonic decays, having reduced performance of the secondary vertex-based taggers. This dedicated tagger combines a set of variables to identify muons in jets and discriminate their primary or secondary origin through a BDT discriminant (see Figure 1) [5, 7]. The typical muon identifiction efficiency in $b$-jets is $65 \%$ for a $1.8 \%$ pion misidentification probability. This results in a tag efficiency of $b$-jets of $\sim 10 \%$ with a light-jet mistag probability of just $0.2 \%$. The adoption of the SMT tagger reduces the drop in $b$ tagging efficiency of the high-level discriminants for $b$-jets containing muons from the $b$-hadron decay chain.
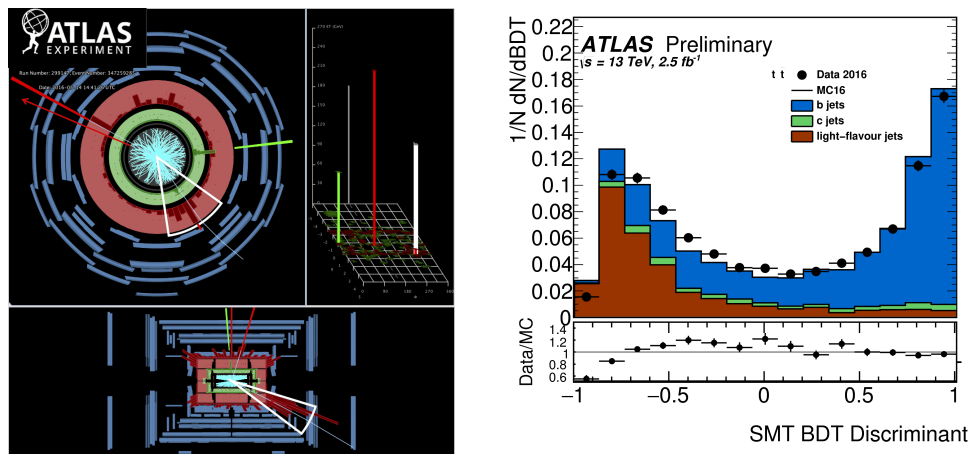

Figure 1: Soft Muon Tagging: (Left) Event display of a $t \bar{t}$ dilepton candidate event from $p p$ collisions recorded by ATLAS at $\sqrt{s}=13 \mathrm{TeV}$ with a soft $(7 \mathrm{GeV})$ muon associated to a $b$-jet candidate tagged by the new SMT tagger. The light blue lines reaching the Muon Spectrometer (MS) show the path of the two combined muons. (Right) Data-MC comparisons of the SMT BDT output for a $t \bar{t}$-dominated $e \mu$ sample. The contributions of $b$-, $c$ - and light-jets in simulation are shown (from [5]).

Vertex-based taggers in ATLAS use both an inclusive secondary vertex approach (SV1) [8] and a topological decay reconstruction (JetFitter) [9, 10]. In the first case, the algorithm starts from twotrack vertices reconstructed using tracks fulfilling strict quality criteria, drops those consistent with $K_{S}^{0}$ or $\Lambda^{0}$ decays and material interactions and merges the selected vertices into a single inclusive secondary vertex. The topological JetFitter algorithm reconstructs the heavy hadron decay chain by applying a modified Kalman Filter formalism, where the distance from the track crossing with jet axis replaces that from the vertex position in the minimisation process. This procedure reconstructs 
the secondary and tertiary vertex in the decay chain, including those with single prongs. The two vertexing algorithms are complementary in their efficiency, with up to $15 \%$ of reconstructed vertices in $b$-jets found by only one of the two methods. The topological reconstruction of $b$ hadron decays is particularly useful to efficiently and cleanly separate $b$ - from $c$ - and light-jets. Detailed information on the reconstructed topology and kinematics of detached vertices offers clear advantages in the rejection of non- $b$ jets, with gains of order of a factor of 2 compared to the use of more inclusive decay properties. The distribution of the invariant mass of reconstructed for jets with a single reconstructed vertex and the full topology of secondary and tertiary vertices, of Figure 2 highlights the purity achievable in $b$-jets in this category at sufficiently large values of the vertex mass.
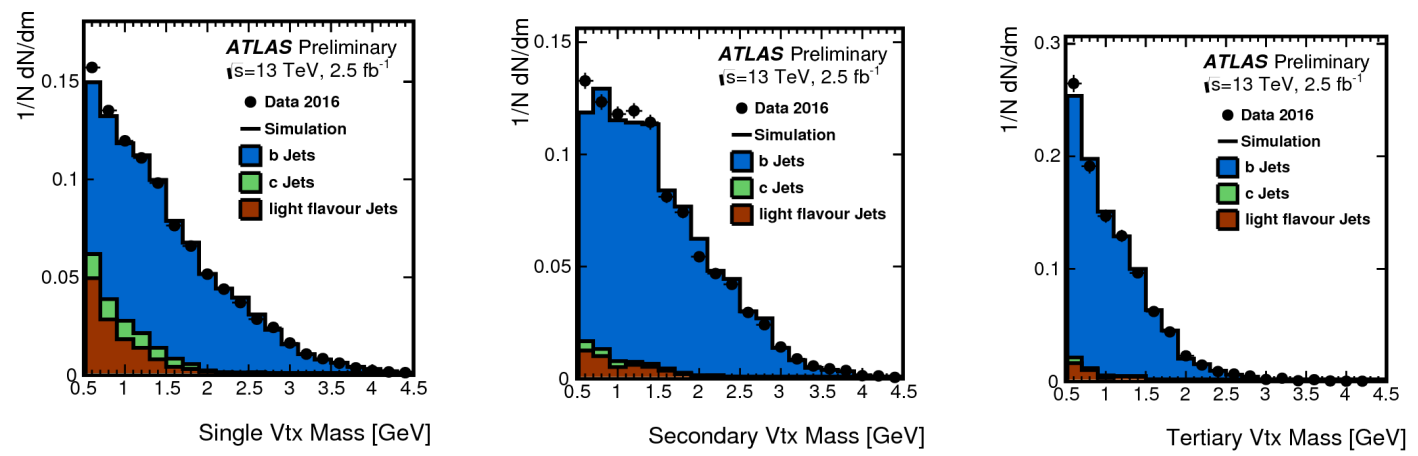

Figure 2: Invariant mass of tracks associated to detached vertices reconstructed by the JetFitter topological vertex reconstruction algorithm in a subset of 2016 collision data and simulated $t \bar{t}$ and $W t$ events for jets with single reconstructed vertex (left), and for secondary (center) and tertiary vertices (right) in jets with two reconstructed vertices. The contributions of $b$-, $c$ - and light-jets in simulation are shown.

In addition, the topological and kinematical properties of decay products originating at a single displaced secondary vertex reconstructed by JetFitter, are exploited for a dedicated high-level $c$-jet discriminant, for use in analyses such as $H^{0} \rightarrow c \bar{c}$ searches.

\section{High-level Discriminants and Training}

High-level discriminants combine the inputs from track-, particle- and vertex-based physics taggers using multivariate classifiers to maximise the $b$-tagging performance. The 2017 ATLAS configuration adopts two classes of high-level taggers: a BDT discriminant (MV2), an evolution of that adopted in 2016 [11], and the first evaluation of a Deep Learning Neural Network discriminant (DL1). Both discriminants have been trained on three sets of variables: i) the variables related to the track-based impact parameter and the vertex-based physics taggers already used in 2016 [11], ii) those with the addition of the particle-based SMT BDT and iii) those with the further addition of the RNNIP track-based tagger.

The relation between the $p_{\mathrm{T}}$ and energy of the jet to those of the original $b$-quark and hadron is key for understanding the flavour tagging response as a function of the jet $p_{\mathrm{T}}$. In $t \bar{t}$ events, used in the past for the training of the high-level discriminants, $b$-jets originate from the relatively lowmass top quark. This results in small $p_{\mathrm{T}}$ transferred to the heavy $b$-hadron and leads to a correlation 
between jet $p_{\mathrm{T}}$ and heavy hadron $p_{\mathrm{T}}$ only for $p_{\mathrm{T}} \lesssim m_{t}$. For jet $p_{\mathrm{T}} \gtrsim m_{t}$, the jet transverse momentum is determined by nearby hadronic activity unrelated to the heavy hadron and the correlation is therefore reduced. Instead, $b$-hadrons produced in the decays of massive particles, such as $Z^{\prime}$ decays, have a high degree of correlation between the hadron $p_{\mathrm{T}}$ and the jet $p_{\mathrm{T}}$ up to large values. In order to include both these regimes in the training of the discriminants, a new hybrid sample has been introduced. The sample is made of $t \bar{t}$ events, to characterise the $b$-hadron $p_{\mathrm{T}}$ region below $250 \mathrm{GeV}$, and decays of a broad $Z^{\prime}$ resonance, to probe the high $p_{\mathrm{T}}$ regime [12].

The development of the discriminant based on the Deep Learning architecture is motivated by the interest in assessing whether this architecture might be better suited to exploit input correlations than a BDT [13]. At present, the performance observed for the DL and BDT discriminants is found to be very similar and these algorithms are also found to tag a highly correlated sample of jets, when trained on the same input variables. In future, DL1 may provide specific advantages when combining large sets of correlated variables, enable an higher degree of integration of low-level and high-level taggers and the adoption of new training procedures to mitigate the impact of modelling systematics.

\section{Performance, Data/MC Response and Calibration}

The performance of the MV2 discriminants in terms of light- and $c$-jet rejection for constant $77 \% b$-jet efficiency as a function of the jet $p_{\mathrm{T}}$ is summarised in Figure 3, comparing to the results obtained for the MV2c10 discriminant in the 2016 configuration [11], and the light-jet rejection is evaluated as a function of the $b$-jet efficiency in Figure 4. The MV2 taggers trained on the hybrid sample achieve a performance similar to those trained on the $t \bar{t}$ sample for jet $p_{\mathrm{T}}$ values below $250 \mathrm{GeV}$ but they outperform the $t \bar{t}$ training by a factor of more than 2 at $77 \%$ b-jet efficiency for $p_{\mathrm{T}}>250 \mathrm{GeV}$. The addition of the SMT information in the MV2Mu discriminant improves
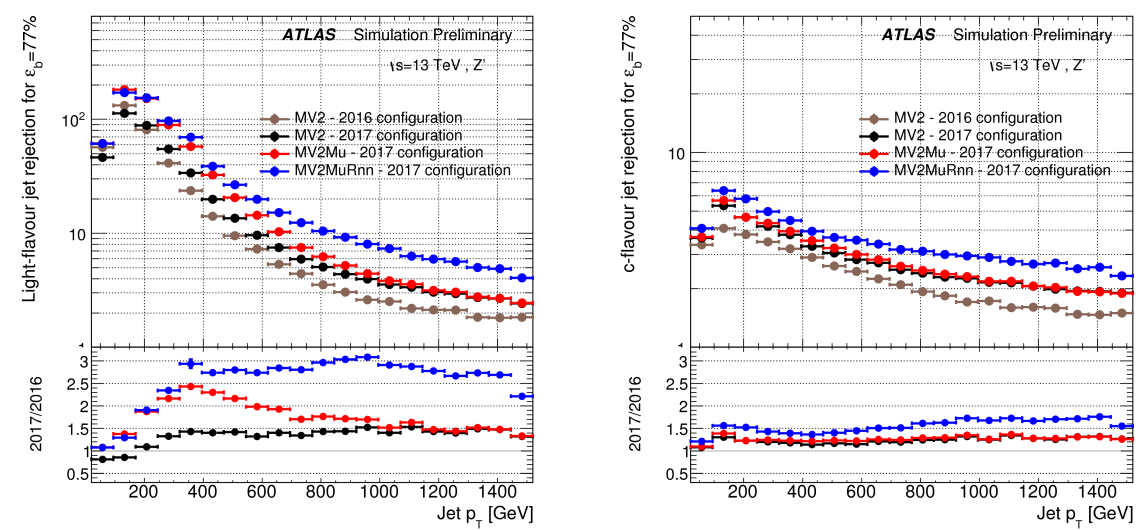

Figure 3: Light-flavour (a) and c-jet (b) rejection as a function of the jet $p_{\mathrm{T}}$ for MV2c10 in the 2016 configuration (brown markers), MV2 (black markers) in the 2017 configuration, MV2Mu (red markers) and MV2MuRnn (blue markers). The algorithm evaluation is performed on $Z^{\prime}$ events for a flat b-jet efficiency of $77 \%$ for each $p_{\mathrm{T}}$ bin. The ratio is calculated with respect to the 2016 configuration (from [5]).

the light-jet rejection in the low-to-medium jet $p_{\mathrm{T}}$ range. The MV2MuRnn setup provides further 
improvements in light- and $c$-jet rejection, in particular in the high- $p_{\mathrm{T}}$ region where the use of the RNNIP tagger is most effective.
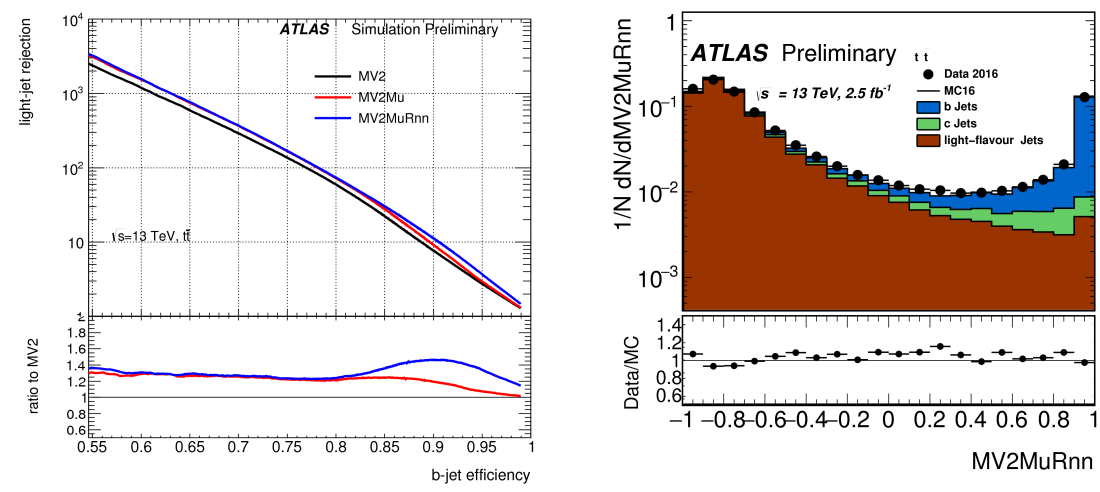

Figure 4: High-level MV2 discriminant: (left) Light-flavour rejection as a function of $b$-jet efficiency for MV2 (black line), MV2Mu (red line) and MV2MuRnn (blue line). The algorithm evaluation is performed on $t \bar{t}$ events. The ratio on the lower panel of the figure is obtained for each MV2 variant (MV2Mu, MV2MuRnn) with respect to MV2. (right) Data-MC comparison of the response of the MV2MuRNN b-tagging algorithm using a selected $t \bar{t}$-dominated $e \mu$ sample. The contributions of $b-, c$ - and light-jets in simulation are shown (from [5]).

In addition to the improvements in the algorithms employed, the optimisation of the flavour tagging response has included detailed comparisons of 2016 collision data and Monte Carlo simulation from track variables to physics tagger observables and high-level discriminant response for event samples enriched and depleted in $b$-jets (see Figure 4).

The stability of the baseline MV2 discriminant efficiency on physics signal samples of different kinematics, $\tilde{t}$ and $\tilde{b}$ pair production and $V H, H \rightarrow b \bar{b}$, has been verified. In particular, in the associated production of the Higgs boson with vector boson $(V H)$ at $m_{H}=125 \mathrm{GeV}$, signal $b$-jets produced in the $H \rightarrow b \bar{b}$ decay and background $Z+$ jets and $W+$ jets events, without $b$-jets, have been considered. After event selection, the ratio of the selected $b$-jets in the $V H$ signal sample to those measured in the hybrid sample is found to be $1.03 \pm 0.02$ (stat.) inclusively in $p_{\mathrm{T}}$, for the MV2MuRnn discriminant at a working point corresponding to $77 \%$ efficiency. The misidentification probability of $c$ - and light-flavour jets in $Z+$ jets and $W+$ jets events fulfilling the same selection as the signal sample are found to be compatible to those observed in the hybrid sample in the same $p_{\mathrm{T}}$ range, for both $c$ - and light-flavour jets.

It is essential not only to obtain a performant high-level discriminant, but also one with wellunderstood performance and small sensitivity to decay modelling systematics. The sensitivity to pile-up conditions and modelling of $b$-production and decay have been characterised [5]. A detailed calibration of the performance of the discriminants is performed comparing the response on simulated and real data and computing appropriate MC scaling factors as a function of the jet $p_{\mathrm{T}}$ and $\eta$. The data-to-MC scale factors extracted in the calibration are used as corrections to the simulation for analyses relying on jet flavour tagging. The results summarised here, obtained for the MV2c10 BDT discriminant in the 2016 ATLAS configuration [11], are exemplificative of the level of accuracy achieved in the flavour tagging calibration. 
The $b$-jet tagging efficiency is extracted using a tag-and-probe (T\&P) [14] and a combinatorial likelihood [15] technique for $t \bar{t}$ di-lepton events. The three main parameters influencing the flavour tagging performance are the jet $p_{\mathrm{T}}$ and $\eta$ and the overlap between the $b$-parton and additional hadronic activity (see Figure 5). This additional hadronic activity may be due to a nearby hadronic jet, at low to intermediate jet $p_{\mathrm{T}}$ values, or gluon radiation within the jet, more notable for high $p_{\mathrm{T}}$ jets. The observed MC scaling factors are typically $\leq 5 \%$.
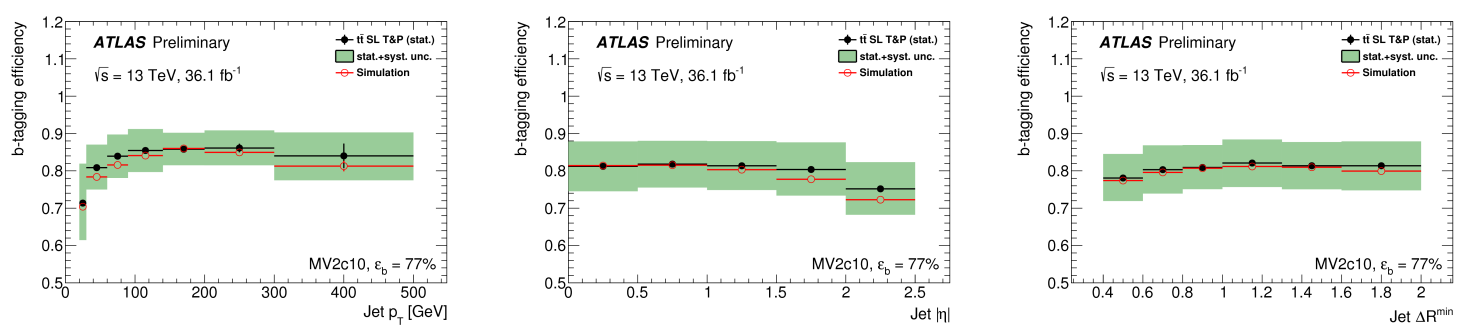

Figure 5: $b$-tagging efficiency for the MV2 algorithm at $77 \%$ efficiency working point as a function of the jet $p_{\mathrm{T}}$ (left), $|\eta|$ (center) and $\Delta R$ to the closest jet (right) obtained selecting $t \bar{t}$ single-lepton events. The red line shows the $b$-tagging efficiency predicted by simulation and the black dots the values measured in data with a T\&P method. The vertical error bars indicate the statistical uncertainty on the measurement. The green band indicates the total statistical and systematic uncertainties.

The mis-identification of light-jets is due to several sources including: resolution effects, material interactions and long-lived particles. An accurate material mapping performed using reconstructed hadronic interaction vertices provides more reliable modelling of the material responsible for hadronic interaction [16].

The effect of the track extrapolation resolution can be estimated by studying simulation adjusted to reproduce the measured impact parameter and taggers fed with tracks with negative lifetime-signed impact parameters [17]. Results for the negative tag measurements, including simulation-based corrections for heavy flavour jet contamination and light-flavour jets with true secondary vertices, are shown in Figure 6.
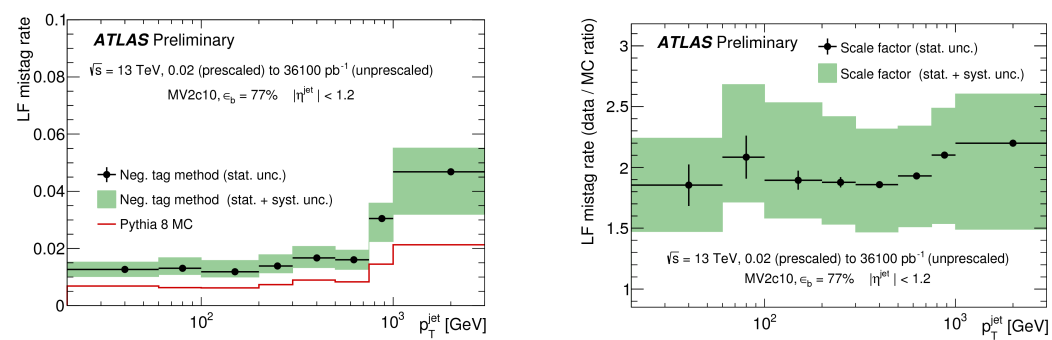

Figure 6: Light-flavour mistag rate (left) and ratio (right) between the light-flavour mistag rate measured from data and that from simulation for the $77 \% b$-efficiency working point as a function of the jet $p_{\mathrm{T}}$, measured by the negative tag method compared to simulation predictions. The statistical uncertainty is the quadratic sum of data and MC statistical uncertainties.

Finally, the effects of uncertainties in $b$-modelling on the predicted $b$-jet efficiency are found to be small compared to the typical uncertainties of calibration analyses in flavour-enriched control 
regions.

\section{References}

[1] ATLAS Collaboration, JINST 3 (2008) S08003.

[2] ATLAS Collaboration, CERN-LHCC-2010-013, ATLAS-TDR-19.

[3] H. Bichsel, Rev. Mod. Phys. 60 (1988) 663.

[4] ATLAS Collaboration, JINST 11 (2016) no.04, P04008 [arXiv:1512.01094 [hep-ex]].

[5] ATLAS Collaboration, Note ATL-PHYS-PUB-2017-013.

[6] ATLAS Collaboration, Note ATL-PHYS-PUB-2017-003.

[7] A. Sciandra, in these proceedings.

[8] ATLAS Collaboration, Note ATL-PHYS-PUB-2017-011.

[9] G. Piacquadio and C. Weiser, J. Phys. Conf. Ser. 119 (2008) 032032.

[10] G. Gilles, in these proceedings.

[11] ATLAS Collaboration, Note ATL-PHYS-PUB-2016-012.

[12] F. Di Bello, in these proceedings.

[13] M. Lanfermann, in these proceedings.

[14] ATLAS Collaboration, Note ATLAS-CONF-2016-001.

[15] ATLAS Collaboration, Note ATLAS-CONF-2014-004.

[16] ATLAS Collaboration, JINST 11 (2016) no.11, P11020 [arXiv:1609.04305 [hep-ex]].

[17] ATLAS Collaboration, Note ATLAS-CONF-2014-046. 